\title{
Buckling of Rectangular Isolated R.C. Columns: Closed-form Approxima- tion for Interaction Domains
}

\author{
Domenico Raffaele, Giuseppina Uva, Francesco Porco and Andrea Fiore*
}

DICATECh; Politecnico di Bari, Via Orabona 4- 70126 Bari, Italy

\begin{abstract}
Interaction domains for the buckling of isolated R.C. columns are an efficient and versatile instrument for the assessment of the resistance at Ultimate Limit State, and allow the optimization of the structural geometry and reinforcement ratio.

The paper presents the procedure for deriving interaction domains for rectangular symmetrically reinforced columns, providing a detailed analysis of the load-carrying capacity for various classes of concrete and reinforcement steel bars. Domains have been obtained according to the "model-column method", taking into account the uncertainties both in geometry and in the position of axial loads. Effects related to short-term creep are ignored.

In order to facilitate the practical utilization, the generic domain has been approximated by a two-branch curve, parabolic and elliptic. The first-one is related to the collapse dominated by axial load, and the second one to flexural crisis. This approximation leads to simple closed-form expressions, particularly suitable for engineering preliminary design.
\end{abstract}

Keywords: Buckling, isolated R.C. columns, interaction domains, reinforced concrete, axial force, closed-form solution.

\section{INTRODUCTION}

In RC framed buildings, columns are the most important primary structural elements, and have a crucial role both under vertical and seismic loads. A column is typically subjected to axial force and bending moment which can be induced by the continuity with the beams and/or by the eccentricity due to the imperfect alignment between beam and column. The cross section is designed in order to guarantee that the actions are compatible with the resisting capacity, represented by the $\mathrm{N}-\mathrm{M}$ (axial force-bending moment) interaction diagram.

In the last few decades, the use of slender RC columns has become more and more widespread in civil buildings and infrastructures (especially bridges). This is often the consequence of architectural and aesthetic needs, but also of the availability of high strength materials, that has boosted the effort towards the structural optimization. Obviously, the collapse of slender RC columns is not strictly dependent on the strength of the section, but can be driven by instability phenomena, and this would require that the numerical modelling is particularly attentive and rigorous, including among other aspects - second order effects. As a significant example, it is worth mentioning the case of bridge piers, which are a typical example of structures particularly sensitive to buckling, and for which a number of research studies and applications have been developed [1].

It is evident that the availability of simplified procedures for the qualitative assessment of materials $[2,3]$ and for the

*Address correspondence to this author at the DICATECh; Politecnico di Bari, Via Orabona 4 - 70126 Bari, Italy; Tel: +39 080 5963832;

Fax: +39 0805963823; Email: andrea.fiore@ poliba.it seismic assessment can be very helpful in order to reduce the computational burden and allows an effective management of engineering problems.

In the literature, several research studies about the behaviour and design of slender RC columns can be retrieved. Bazant et al. [4, 5] analytically calculated the resistance capacity of slender RC columns on the basis of the balance equation and the strain compatibility condition, by assuming a deflection curve with a sinusoidal function. Kim et al. [6,7] proposed a numerical method that considers the material and geometric nonlinearities. Yalcin and Saatcioglu [8] developed an analytical model which considers the influence of lap splice failure and the length of the plastic hinge on the nonlinear behaviour of RC columns.

Most building codes, such as Eurocode 2 [9] and ACI318 [10], and technical standards, in order to guarantee an adequate versatility, provide the indications about the column resistance independently from the slenderness ratio and then take into account the possible instability effects in "long" columns by increasing the applied first-order moment. The increase is based on the moment magnification factor derived from elastic stability theory and the method is called "Magnified Moment Method". Its accuracy depends on the effective flexural stiffness EI of the column, which is influenced by cracking, creep and non-linear behaviour of the material. Over the last three decades, many authors and national codes have proposed different methods to determine the column stiffness for short and long-term loads. Eurocode 2, ACI-318 and most authors, such as Mirza et al. (1987), Mirza (1990), Bonet et al. (2004), Tikka and Mirza (2005 and 2008)[11-15], claim that the flexural stiffness EI depends on the loads applied by means of the relative eccentricity or else through the axial load. In a recent study by 
Bonet et al. (2011) [16], the different formulations EI proposed in the technical literature and in the technical codes are discussed and compared, showing that there is a great variability and dispersion of the different proposal with regard to the variables involved and the functions used. Consequently, the application of different technical codes may result in the underestimation of the resistance, which is more accentuated if the slenderness increases.

It is evident that the construction of reliable interaction domains for RC columns characterized by high slenderness ratio is fundamental, but requires a rigorous analysis (because of nonlinearities due to cracking and $2^{\text {nd }}$ order effects) which is time consuming, and whose use in design practice requires much experience [17].

This paper presents a simplified analytical model for assessing the resistance of isolated RC rectangular columns. The model takes into account material and geometric nonlinearities, and a graphical approach is adopted to define the interaction domains at the Ultimate Limit State. These domains are built by means of dimensionless parameters that define the different parts of the domain. The aim of the study is to define an efficient tool that can be used for the simplified, preliminary design/assessment of the structural geometry and of the reinforcement ratio of isolated columns.

It should be noted that the proposed simplified model is reliable in all those cases in which the static and geometric assumptions are coherent with the "model column method" [9]. The entire proposed model can be easily automated in order to allow an immediate use.

\section{THEORETICAL BASIS}

The cantilever column shown in (Fig. 1) is the simplified model widely used in the literature in order to assess buckling effects. A similar model has been recently studied by Krauberger et al. (2011) [18] for an ideal RC column without any imperfection. However, in the presence of softening induced by the micro-cracking in the concrete, the buckling load of the perfect column may significantly overestimate the limit load of the imperfect column (where the terms, perfect and imperfect, identify, respectively, the column without and with cracking). In order to account for the effects caused by micro-cracking, an additional eccentricity of the axial load must be introduced.

Let us consider an isolated column (Fig. 1a) having constant section and reinforcement along its longitudinal axis. At the generic abscissa $\mathrm{z}$, the balance equation between internal and external actions is given by Eq. 1:

$$
E I y^{\prime \prime}(z)=P e_{0}+P[a-y(z)]
$$

If the boundary conditions are known, according to the Eulerian theory, the elastic deformation is obtained by integrating Eq. 1 twice:

$$
\begin{aligned}
& \alpha=\sqrt{\frac{P}{E I}} \\
& y(z)=\frac{e_{0}}{\cos \alpha L}(1-\cos \alpha z)
\end{aligned}
$$

By introducing the mechanical slenderness $\lambda$, the moment $M_{0}$ at the base section $(\mathrm{z}=0)$ of the column is given below:

$$
M_{0}=\frac{P e_{0}}{\cos \left(\frac{\lambda}{2} \sqrt{\frac{P}{E A}}\right)}
$$

The instability collapse of the column is driven by the collapse of the most stressed section (i.e. the base section). After defining the corresponding resistance domain at the Ultimate Limit State (Fig. 1b), for an assigned axial stress, the maximum load $\left(P_{u}^{*}\right)$ that the column can support can be easily evaluated.

In the (Fig. 1b), point $\mathrm{A}$ identifies the generic ultimate limit load $P_{u}{ }^{*}$ and ultimate bending moment $M_{u}{ }^{*}$ that cause the collapse of the column. The moment value consists of two different contributions:

$$
\text { the first order moment } M_{I}=P_{u}^{*} e_{0}
$$

the second order moment $M_{I I}$ given by the following equation:

$$
M_{I I}=M_{u}-M_{I}=P_{u}^{*} e_{0}\left(\frac{1}{\cos \left(\frac{\lambda}{2} \sqrt{\frac{P_{u}^{*}}{E A}}\right)}-1\right)
$$

The balance equation (Eq. 1) takes into account only the geometrical non-linearity, because the material is considered as perfectly elastic (i.e. the flexural stiffness $E I$ is constant). The presence of mechanical non-linearity induces, instead, variations in the first member of Eq. 1 (internal moment) because the bending stiffness $E I$ changes with stress and curvature. In this case, the problem can be numerically solved, by assuming that the moment/curvature law, under the actual axial load $P$, is known.

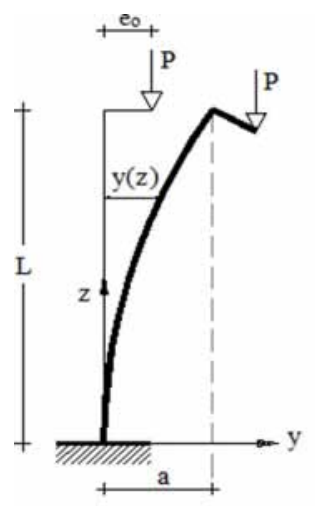

(a)

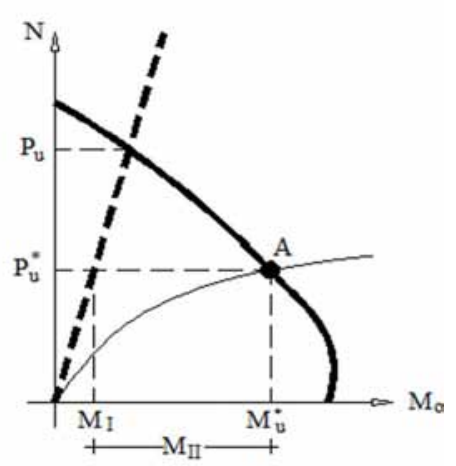

(b)
Fig. (1). Structural scheme of the model column (a) and resistance domain for the base section (b).

If the exact solution of the problem is desired, a more general method should be used. For example, the column shall be divided into a convenient number of sub-elements and then iterative procedures based on finite difference method will be applied. Although conceptually simple, this approach is very laborious because it requires - for each section - the knowledge of the moment/curvature diagram and of the longitudinal actions (and, consequently, the evaluation of the curvature on the basis of the bending moment assumed in the iterative step). In the case of statically determinate elements with a constant cross section, loaded by steady longitudinal forces (with no limitation for other external ac- 
tions), the "model column method" is often used and it is also allowed by Eurocode 2 [9].

In this method, it is assumed in advance that the deformed configuration of the element (Fig. 1a) is a sinusoidal semi-wave of undetermined amplitude:

$$
y(z)=a \cdot\left(1-\cos \frac{\pi z}{2 L}\right)
$$

After deriving equation (Eq. 7) twice, the following expression of the curvature is obtained:

$$
y^{\prime \prime}(z)=a \cdot \frac{\pi^{2}}{4 L^{2}} \cos \frac{\pi z}{2 L}
$$

The effective buckling length is equal to $2 \mathrm{~L}$. Therefore, for $z=0$ (base section, indicated by the subscript " 0 "), the curvature $y^{\prime \prime}(z)=\chi_{0}$ is given by:

$$
\chi_{0}=a \cdot \frac{\pi^{2}}{4 L^{2}} \Rightarrow a=\frac{4 L^{2}}{\pi^{2}} \cdot \chi_{0}
$$

This equation can be expressed as a function of the slenderness $(\lambda=2 L / i)$ :

$$
a=\left(\frac{\lambda \cdot i}{\pi}\right)^{2} \cdot \chi_{0}
$$

Eq. 10 represents a linear relationship between the curvature $\chi_{0}$ and the deflection amplitude $a$ at the top of the column. In restrained section, the balance equation can be written as follows:

$$
M=M_{I}+M_{I I}=M_{I}+P \cdot \frac{\lambda^{2} \cdot i^{2}}{\pi^{2}} \chi_{0}
$$

In Fig. (2), the plot of Eq. 11 in the moment-curvature plane is shown for the base section, for a fixed value of the buckling force $(P)$. The points A and B in which it intersects the curve $M^{I N T}\left(\chi_{0}\right)$, defining the internal moment of the base section, identify the balance conditions. In detail:

- Point A identifies a stable balance condition, since any increase in the curvature would bring a greater increase in the internal moment $M^{I N T}\left(\chi_{0}\right)$.

- Point B identifies an unstable balance condition, since any increase in the curvature would bring a greater increase in the external moment $M^{E S T}\left(\chi_{0}\right)$.

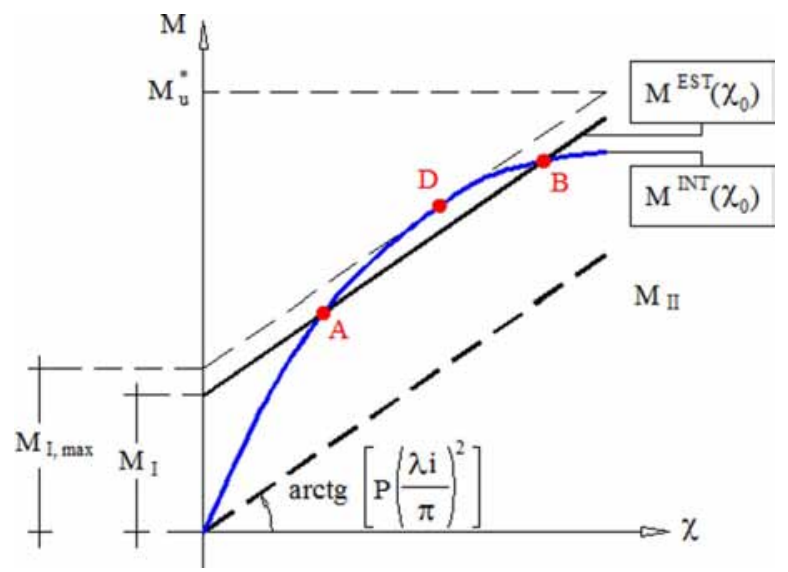

Fig. (2). Moment/curvature diagram of the base section and identification of maximum first order moment at the limit condition of stable balance.
For the base section, the maximum first order moment $M_{I \max }$ is given by the intersection of the tangent to the curve $M^{I N T}\left(\chi_{0}\right)$ and the vertical axis. The tangent is parallel to $M_{I I}$, which is the moment that represents the variation of the second order moment according to the curvature of the section. If other conditions do not change, $M_{I I}$ only varies with the slenderness $\lambda$. Therefore $M_{I, \max }$ is also called: "reduced flexural resistance" of the analysed structure. The point D represents the limit condition of stable balance. The couple $M_{I}-$ $P$, just obtained, represents one limit point of the "reduced resistance domain" of the examined column for an assigned value of the slenderness $\lambda$.

Although it is conceptually simple to define, the construction of the "reduced resistance domain" presents some numerical difficulties, which have been pointed out in the last years by many researchers $[6,8,17]$. The major difficulty lies in the evaluation of the maximum value of the available first order moment $M_{I}$ for a sequence of possible slenderness values of the column, loads $P$ and reinforcement arrangements. These difficulties highlight that the availability of "reduced resistance domains" - even in an approximate form - would actually provide a very effective tool for professionals, in order to immediately solve design problems related to compressed reinforced concrete columns.

\section{REDUCED RESISTANCE DOMAINS}

In order to formulate the equilibrium equation and define the dimensionless domains for columns with rectangular cross-section symmetrically reinforced, the starting assumptions were made and reported in the list below and in (Table 1).

- Cross sections remain plane (Bernoulli-Navier hypothesis), i.e. the strain distribution on any section is linear.

- There is perfect adherence between concrete and steel.

- Sections are subjected to normal stress only.

- Shear deformations are neglected.

- Tension stiffening and concrete tensile strength are neglected.

Table 1. Symbols and Assumptions for the Geometrical and Mechanical Parameters

\begin{tabular}{|l|c|}
\hline $\begin{array}{l}\text { geometrical dimensions of the } \\
\text { rectangular cross-section }\end{array}$ & $b, h$ \\
\hline $\begin{array}{l}\text { Longitudinal reinforcement } \\
\text { (constant and symmetric) }\end{array}$ & $A_{S, \text { bottom }}=A_{S, \text { top }}=A_{S} / 2$ \\
\hline concrete cover & $0.10 h$ \\
\hline mechanical reinforcement ratio & $\omega=\frac{A_{s} \cdot f_{y d}}{b \cdot h \cdot f_{c d}}$ \\
\hline $\begin{array}{l}\text { dimensionless values of axial load } \\
\text { and bending moment }\end{array}$ & $v^{*}=\frac{N}{b \cdot h \cdot f_{c d}} ; \mu^{*}=\frac{M}{b \cdot h^{2} \cdot f_{c d} . .}$ \\
\hline
\end{tabular}

Fig. (3) shows the simplified function (one of the sides of the overall interaction domain) that allows to evaluate the longitudinal reinforcement of the section, once the dimensionless design (subscript "d") stress $v_{d}{ }^{*}$ and moment $\mu_{d}{ }^{*}$ are 


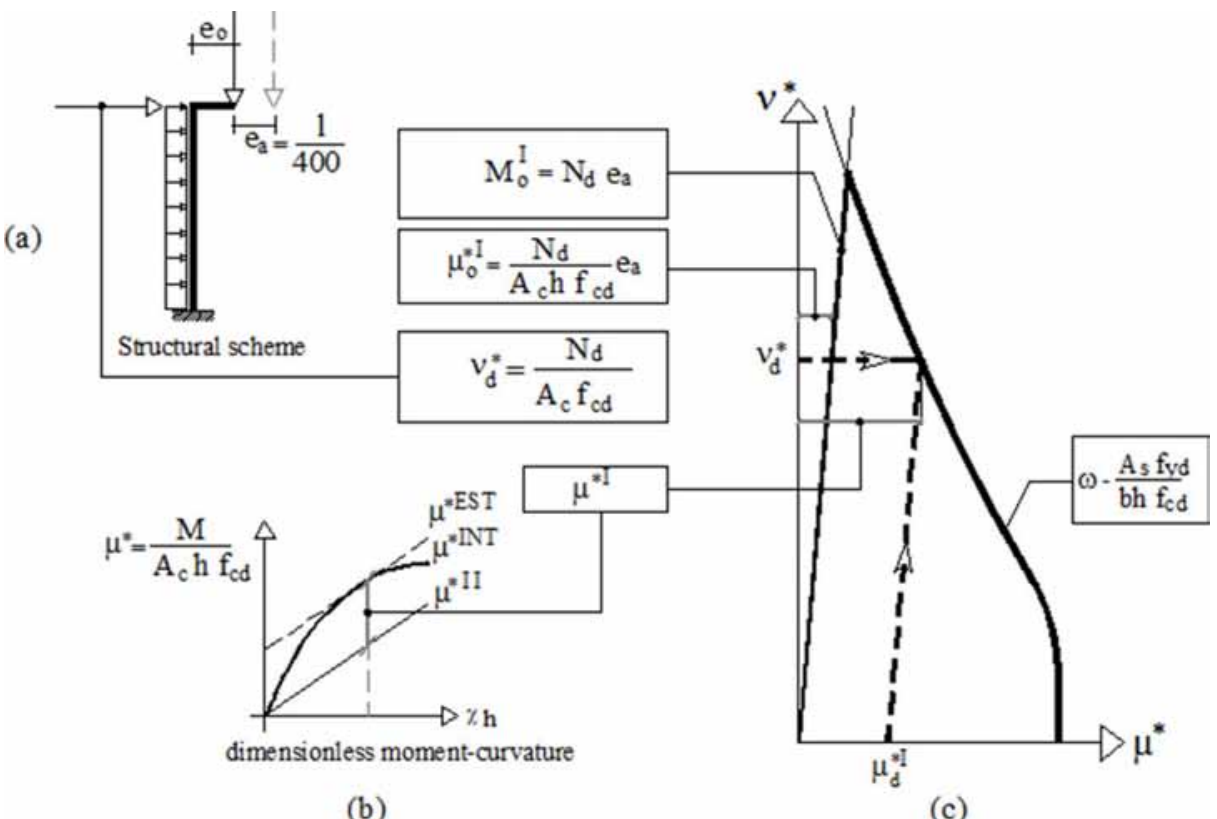

(b)

(c)

Fig. (3). How to use the reduced interaction domains?.

given. The procedure, basically, is the same indicated by Eurocode 2 for isolated columns in braced systems: in order to account for uncertainties (in the geometry and position of axial loads through an additional eccentricity $e_{a}=l_{0} / 400-$ see $\$ 5.2$.(9) in EC2), interaction domains are further reduced by a specific limitation imposed on the first-order moment:

$$
\left(M_{t}\right)_{\min } \geq \frac{1}{400} l_{0} N_{d} \Rightarrow \mu^{*} h \geq \frac{1}{400} l_{0} v^{*} \Rightarrow v^{*} \leq 400 \frac{h}{l_{0}} \mu^{*}=400 \frac{2 \sqrt{3}}{\lambda} \mu^{*}=\frac{800 \sqrt{3}}{\lambda} \mu^{*}
$$

It should be pointed out that the parameter $800 \sqrt{ } 3 / \lambda$ represents the angular coefficient of the lines delimiting the reduced domain, and, additionally, that Eq. 12 is only valid for rectangular sections.

The difference $\mu_{\mathrm{d}}{ }^{*}=\mu{ }^{*}-\mu_{\mathrm{o}}{ }^{*} \mathrm{I}$ shown in (Fig. 4) is the maximum available dimensionless I order moment.

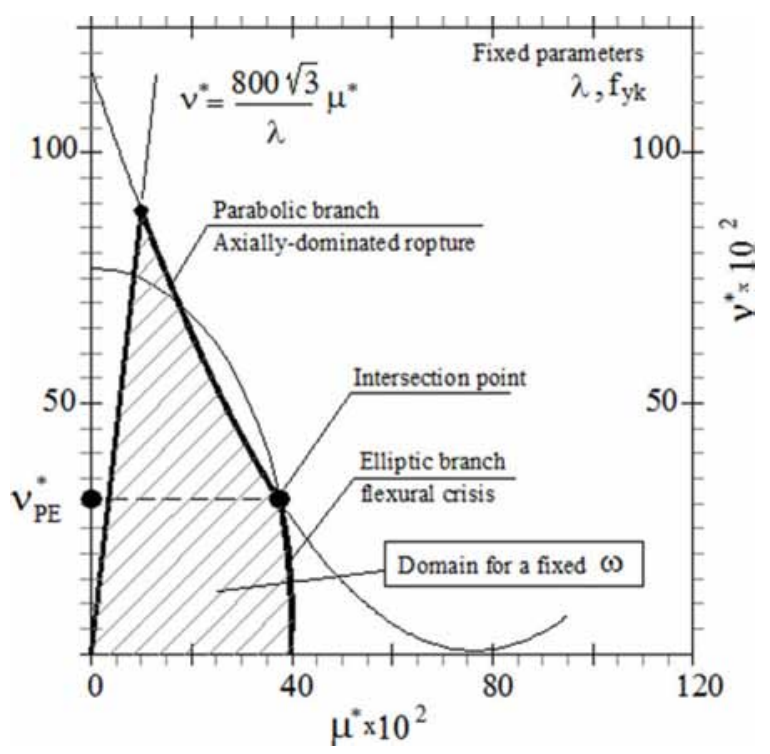

Fig. (4). Simplified interaction domain for an isolated rectangular column (for assigned values of $\omega$ and $\lambda$ ).

\section{CLOSED-FORM APPROXIMATION OF DOMAINS}

As previously mentioned, the practical use of "reduced resistance domains" is often limited because of a number of difficulties and drawbacks:

- Need of interpolating the values of slenderness and reinforcement ratio which are not directly represented in the domains.

- Need of a "graphical determination" of the resistance values.

An analytical formulation of the domains, even approximate, appears therefore particularly interesting for its simplicity and rapid application.

For slenderness values in the range $50<\lambda<150$ and mechanical reinforcement ratios in the range $0.5<\omega<1.5$, the generic interaction domain can be approximated by a twobranch curve: "parabolic" and "elliptic". The first curve represents the case of a prevalently axial collapse and; the second branch is related to the flexural failure of the column (Fig. 4).

The parameter that marks the boundary between the two different branches is $v_{P E}{ }^{*}$, that is, the dimensionless axial limit force. $v_{P E} *$ depend on $\lambda$ and $\omega$. It is defined by analytical expressions that are reported in APPENDIX.

In the following section, the analytical expression of each curve is obtained, taking into account, roughly, the influence of all geometrical and mechanical parameters $\left(\lambda, \omega, f_{y k}\right)$.

Table 2 contains a summary of the parameters involved in the calculation of the parabolic and elliptic branches which approximate the "reduced interaction domain" for isolated columns with rectangular section and symmetrical reinforcement.

$k_{x}=k_{x}(\omega, \lambda)$ and $k_{y}=k_{y}(\omega, \lambda)$ represent the coordinates of the points of the parabolic branch of the domain. Fig. (5a) 
Table 2. Main Parameters for Assessment of Parabolic and Elliptical Branch

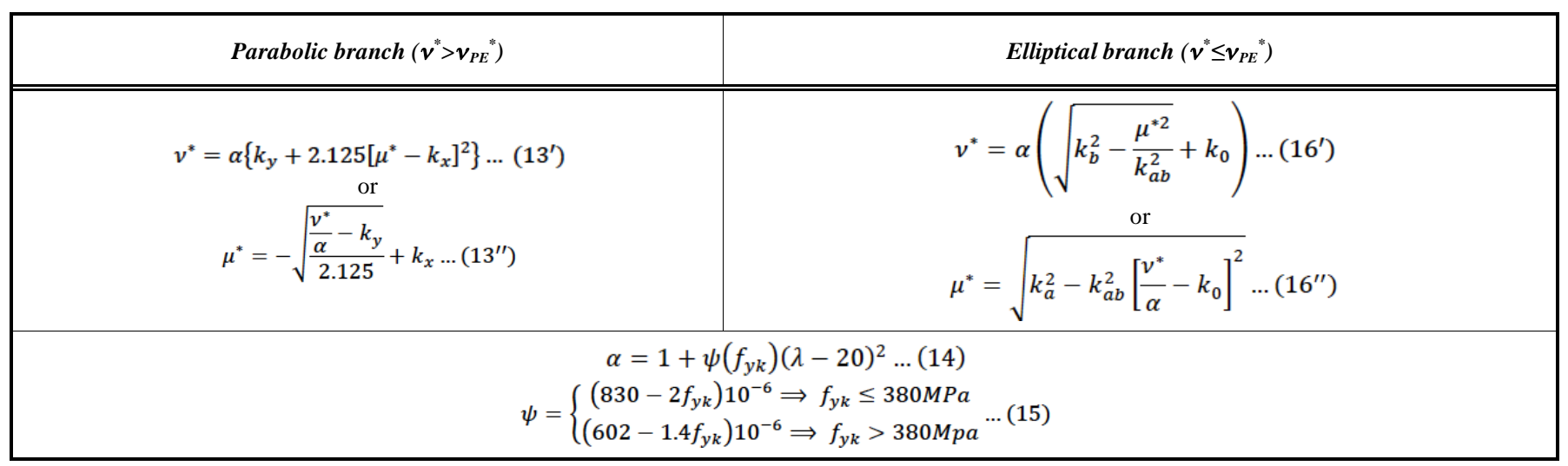

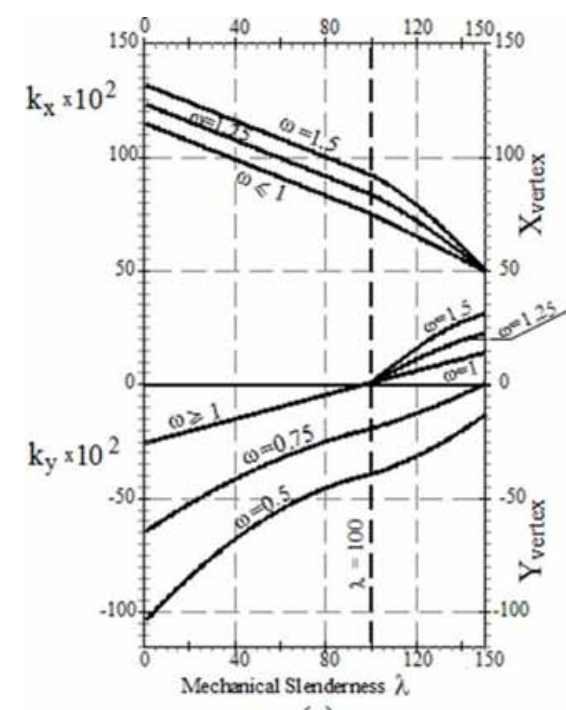

(a)

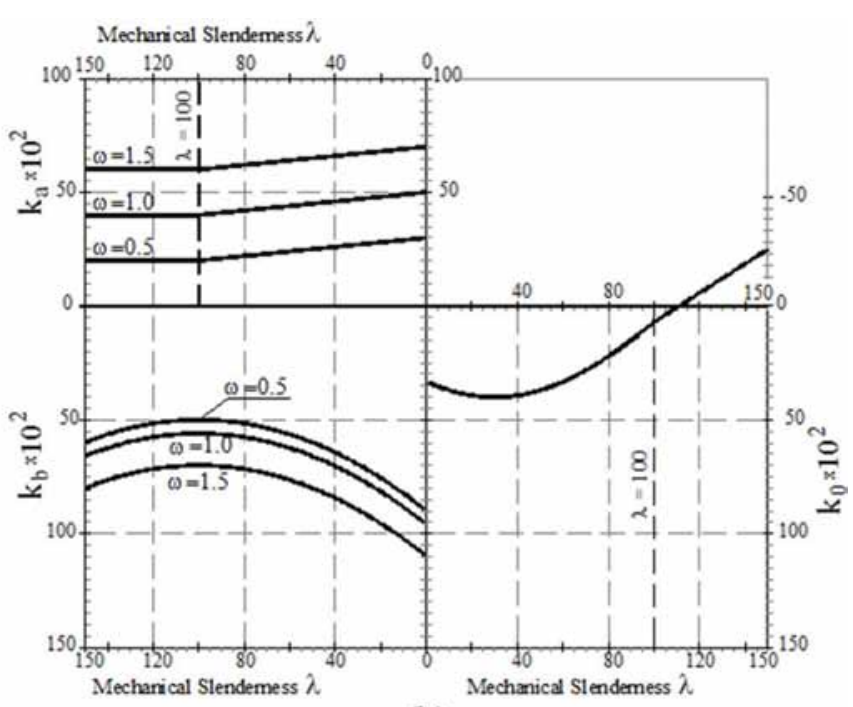

(b)

Fig. (5). Plot of the parameter which defines the parabolic curve (a) and the elliptic curve (b).

shows the plots of $k_{x}$ and $k_{y}$ vs $\omega$ and $\lambda$, whereas their analytical expressions are explicated in APPENDIX.

Fig. (6) reports the function $\alpha=\alpha\left(\lambda, f_{\mathrm{yk}}\right)$. Table 2 reports the analytical expressions of the main parameters defining the parabolic and elliptical branch. Numerical analyses have shown that the coefficient $\alpha$ essentially depends on the slenderness $\lambda$ of the column characteristic yield strength of steel reinforcement $\left(f_{y k}\right)$. Its value can be assumed equal to 1 if the slenderness values are less than 20 .

In Eqs. 16' and 16", $k_{a}=k_{a}(\omega, \lambda)$ and $k_{b}=k_{b}(\omega, \lambda)$ are the values of the semi-axes of the ellipse in the $\mu^{*}$ and $v^{*}$ directions; $k_{a b}=k_{a} / k_{b} ; k_{0}$ is the ordinate of the ellipse center with variation of $\lambda$ (see Fig. 5b and APPENDIX).

The dimensionless axial limit force $v_{P E}{ }^{*}$ marks the transition from the parabolic to the elliptic branch. In a few words, the parabolic branch will be used if ${v_{S d}}^{*} \geq v_{P E}{ }^{*}$, while the elliptic branch will be used if $v_{S d}{ }^{*} \leq v_{P E}{ }^{*}$ (the subscript $S d$ indicates the design value). The analytical expressions of $v_{P E}{ }^{*}$ are reported in the APPENDIX, whereas the plot of the function ${v_{P E}}^{*}(\omega, \lambda)$ is shown in (Fig. 7). It should be noted that the slenderness equal to 100 is a discontinuity point of the function $v_{P E}{ }^{*}=v_{P E}{ }^{*}(\omega, \lambda)$, which is linear for $\lambda<100$, whereas is parabolic for $\lambda>100 v_{P E}{ }^{*}$.

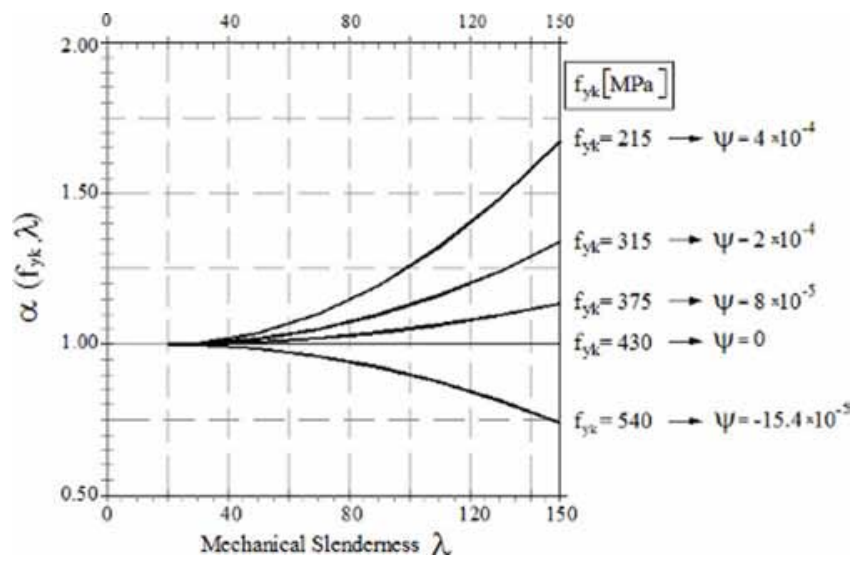

Fig. (6). $\alpha=\alpha\left(f_{y k}, \lambda\right)$ : scaling factor of $v^{*}$.

In Fig. (8), the domains obtained through the "rigorous" approach (black curve) [19](Raffaele, 2004) and the "approximate" method (red curve) are compared.

Domains have been obtained for the steel class $f_{y k}=450 \mathrm{~N} / \mathrm{mm}^{2}$ (450C class of the Eurocode 2 [9] (CEN 2004)). 


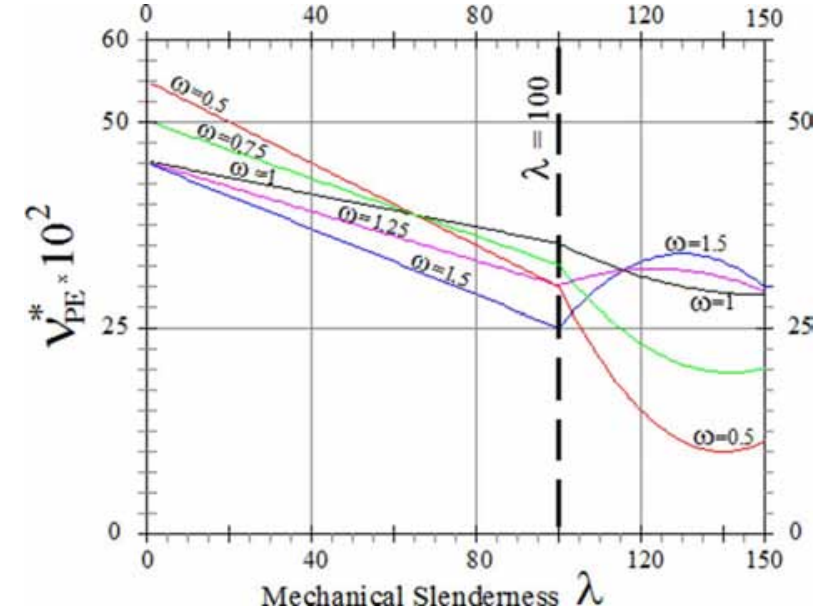

Fig. (7). Plot of the dimensionless axial limit force $v_{P E}{ }^{*}$ as a function of the slenderness $\lambda$.

It is evident from Fig. (8) that the difference between the two approaches is very small for the whole range of variation considered for the slenderness ratio, which is quite large (between 50 and 150 ).

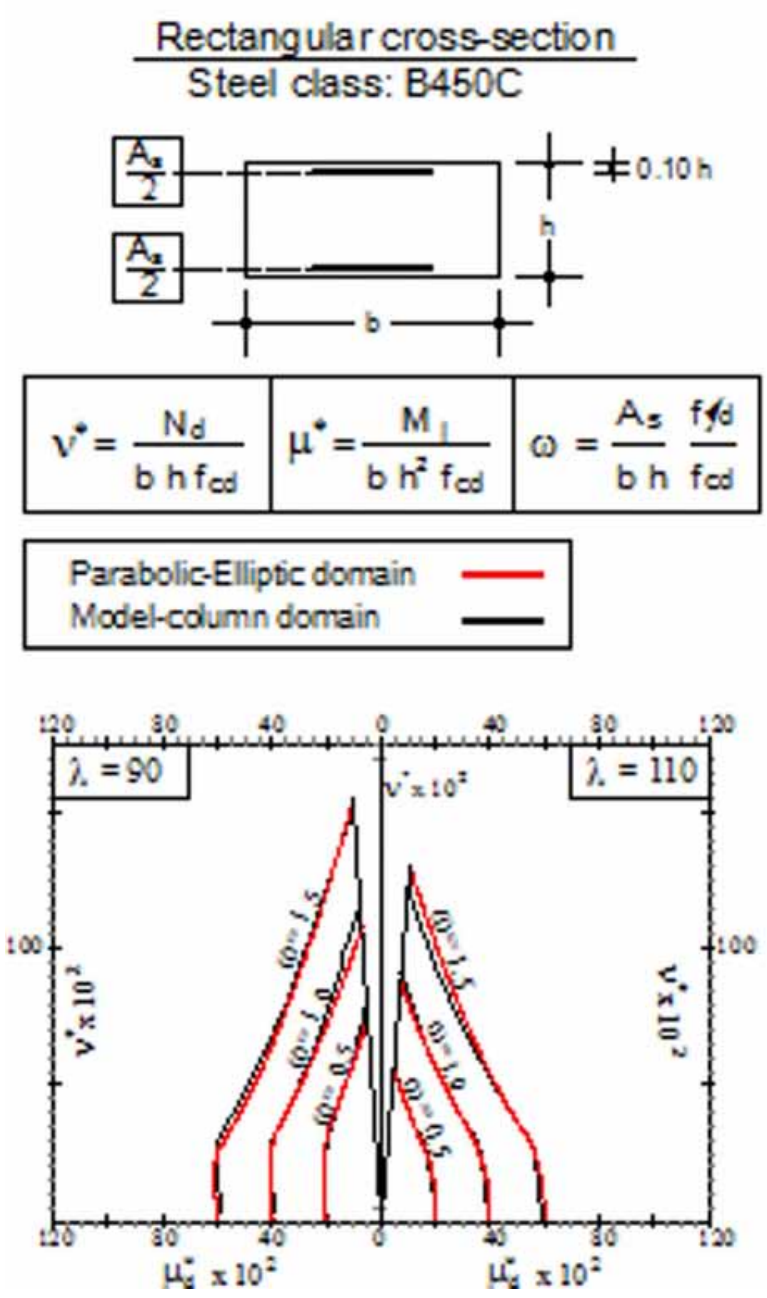

The comparison points out that the proposed simplified analytical approach, based on dimensionless parameters, can be very useful for practical applications and preliminary design, without the need to resort to more refined methods.

\section{NUMERICAL APPLICATIONS}

As an example, the cantilever beam shown in (Fig. 9) is analysed by applying the proposed procedure.

The mechanical parameters used in the examples are as follows:

- Concrete class 28/35: $\mathrm{f}_{\mathrm{cd}}=0.83 \mathrm{R}_{\mathrm{ck}} / \gamma_{\mathrm{c}}=18.16 \mathrm{MPa}$ $\left(\gamma_{\mathrm{c}}=1.5\right)$;

- Steel class B450C [9], $f_{y d}=f_{y k} / \gamma_{s}=391.3 \mathrm{MPa}\left(\gamma_{s}=1.15\right)$.

The mechanical reinforcement ratio assumes the following value:

$$
\omega=\frac{A_{s} f_{y d}}{b h f_{c d}}=\frac{2 \cdot 2260.8 \cdot 391.3}{300 \cdot 1000 \cdot 18.16}=0.31<1
$$

The slenderness $\lambda$ is equal to $2 L / i=104>100(i=8.66 \mathrm{~cm})$.

According to (Eq. 15), the parameters $\psi$ and $\alpha$ are given by $\psi=0$ and $\alpha=1$ for all the slenderness ratios $\lambda$ (see Eq. 14).
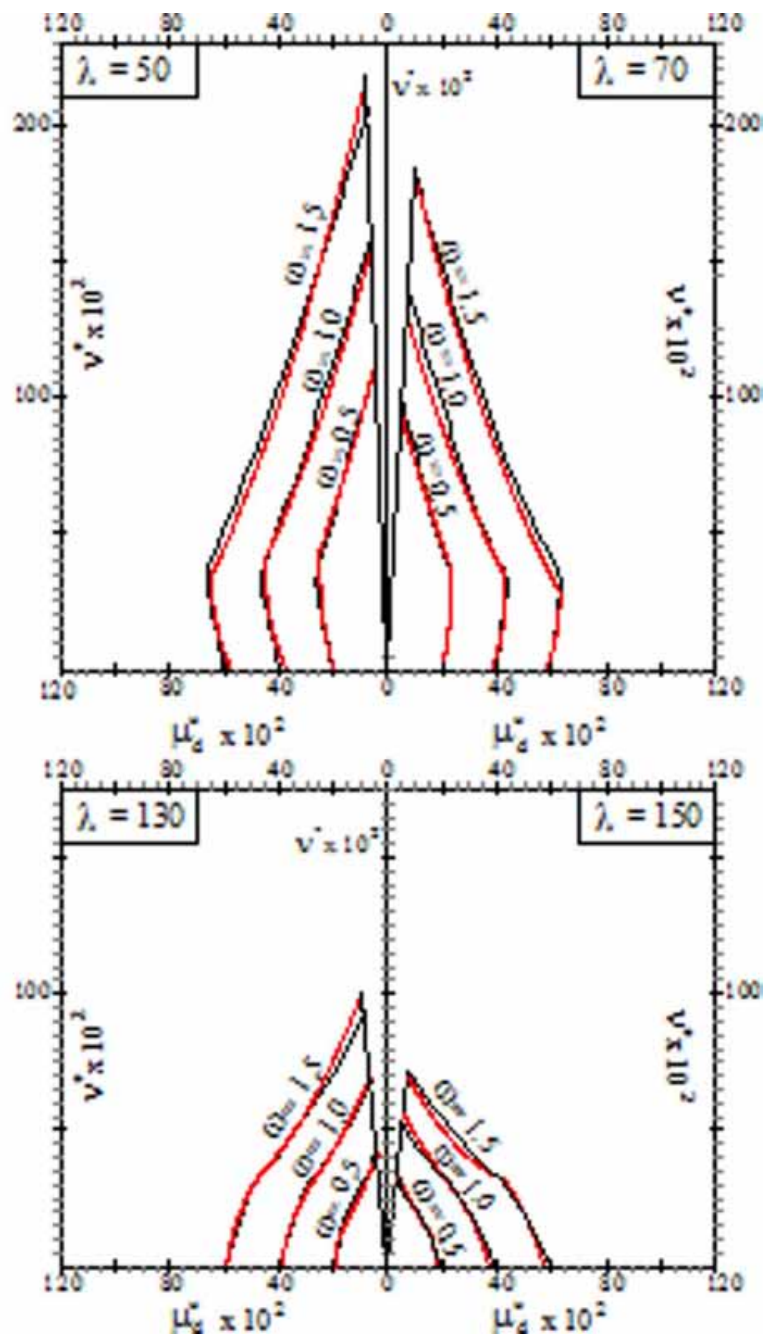

Fig. (8). Interaction domains for columns having rectangular section and symmetric reinforcements: comparison between the rigorous "model-column" method and the approximate parabola-ellipse formulation. 
For $\lambda>100$ and $\omega<1$, it is: $v_{P E}{ }^{*} \times 10^{2}=23.13$ (see Appendix - Eq. A.3).

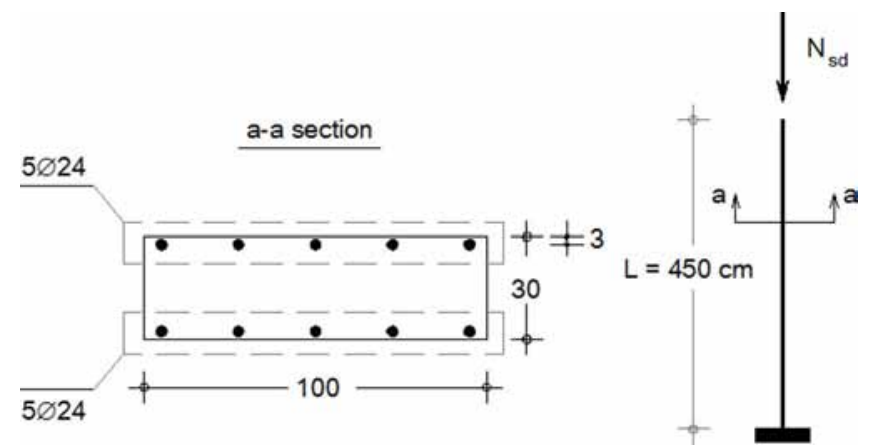

Fig. (9). Geometric data and reinforcement arrangement of the numerical example.

\subsection{Example 1}

As a first example, an axially-dominated collapse is considered: $N_{s d}=2300 \mathrm{kN}$.

In fact, it is obtained:

$$
v_{s d}^{*}=\frac{2300000}{300 \cdot 1000 \cdot 18.16}=42.23 \cdot 10^{-2}>v_{P E}^{*}
$$

In this hypothesis the branch of interest is the parabolic one. The related coefficients are provided by Eqs. A.6, (for $\lambda>100$ and $\omega<1): k_{x}=73 \cdot 10^{-2} ; k_{y}=-53.12 \cdot 10^{-2}$.

Equation 13"' gives:

$$
\mu^{* I}=-\sqrt{\frac{(42.23+53.12) \cdot 10^{-2}}{2.125}}+73 \cdot 10^{-2}=6.01 \cdot 10^{-2}
$$

At this point, the effect of geometric uncertainties is provided by (Eq. 12): $\mu_{0}{ }^{*}=v_{s d}{ }^{*} \lambda 800 \sqrt{ } 3=3.16 \cdot 10^{-2}$.

Eventually, the maximum available I order moment is: $\mu_{d}{ }^{* I}=\mu^{* I}-\mu_{0}{ }^{* I}=2.84 \cdot 10^{-2}$, and $M_{d}{ }^{I}=\mu_{d}{ }^{* I} \cdot b \cdot h^{2} \cdot f_{c d}=46.49 \mathrm{kNm}$.

For the same example, the application of the rigorous "model-column" method gives the value: $M_{d}{ }^{I}=46.33 \mathrm{kNm}$, with a difference lower than $0.5 \%$.

The dimensionless abacus (Fig. 10) provides the value $M_{d}^{I}=45.10 \mathrm{kNm}$, with a difference of about $3 \%$.

\subsection{Example 2}

As a second example, a bending collapse is considered: $N_{s d}=800 \mathrm{kN}$.

$$
v_{s d}^{*}=\frac{800000}{300 \cdot 1000 \cdot 18.16}=14.69 \cdot 10^{-2}<v_{P E}^{*}
$$

Under this hypothesis, the branch of interest is the elliptic one.

The allowable I order-moment is obtained by Eq. 16".

The $k_{a}, k_{b}$ and $k_{0}$ coefficients are provided by Eqs A.10 (for $\lambda>100 ; \omega<1$ ): $\mathrm{k}_{\mathrm{a}}=12.41 \cdot 10^{-2} ; \mathrm{k}_{\mathrm{b}}=47.78 \cdot 10^{-2}$ and

$$
\begin{aligned}
\mathrm{k}_{0}=4.54 \cdot 10^{-2} . \\
\mu^{* I}=\sqrt{\left(12.41 \cdot 10^{-2}\right)^{2}-\frac{\left(12.41 \cdot 10^{-2}\right)^{2}}{\left(47.78 \cdot 10^{-2}\right)^{2}}\left(18.16 \cdot 10^{-2}-4.54 \cdot 10^{-2}\right)^{2}}=12.12 \cdot 10^{-2}
\end{aligned}
$$

The reduction related to the geometric uncertainties, according to Eq. 12, gives: $\mu_{0}{ }^{* I}=v_{s d}{ }^{*} \lambda 800 \sqrt{ } 3=1.10 \cdot 10^{-2}$

and, finally, the maximum available I order moment is: $\mu_{d}{ }^{* I}=\mu^{* I}-\mu_{0}{ }^{* I}=11.02 \cdot 10^{-2}$.

Finally, $\mathrm{M}_{\mathrm{d}}{ }^{\mathrm{I}}=\mu_{\mathrm{d}}{ }^{*} \cdot \mathrm{b} \cdot \mathrm{h}^{2} \cdot \mathrm{f}_{\mathrm{cd}}=180.17 \mathrm{kNm}$.

This value is in a perfect agreement both with the one derived by using the abacus (Fig. 10): $M_{d}{ }^{I}=180.43 \mathrm{kNm}$ and with the one provided by the rigorous "model-column" method: $M_{d}^{I}=179.94 \mathrm{kNm}$, demonstrating the robustness of the proposed procedure.

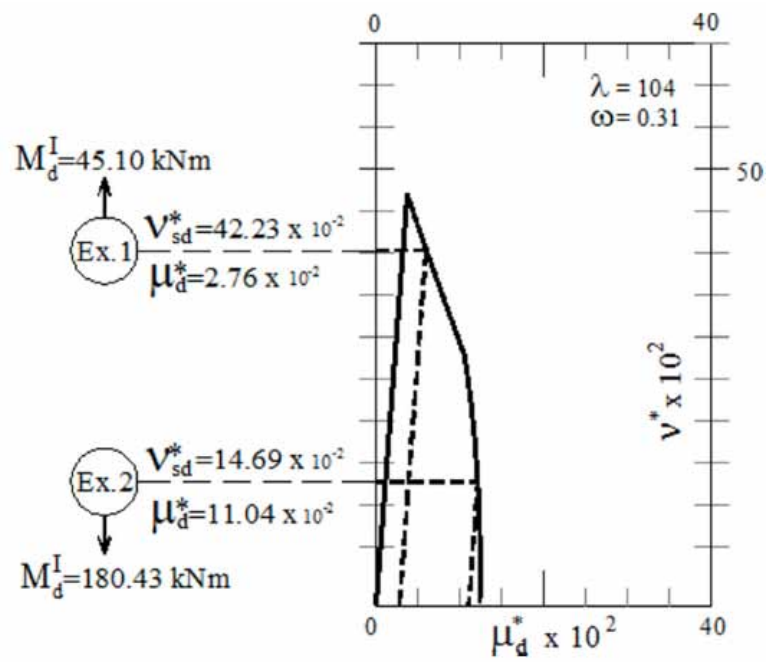

Fig. (10). Plot of the approximated interaction domain of the cantilever column of the examples 1 and 2 .

\section{CONCLUSION}

This paper presents a simple but effective procedure for the design and assessment of slender isolated R.C. columns with rectangular cross section. The proposed procedure is tested by a comparison with results provided by previous studies of the authors [19], based on a numerical approach which simulate the material and geometric nonlinearities through the so called "model-column" method. The formulation derived has shown a good reliability even for a wide excursion of mechanical slenderness and reinforcement ratio.

The essential steps of the procedure can be summarized as follows:

1. Starting from geometric, reinforcement and material data $\left(b, h, L, A_{S}, f_{c k}, f_{y k}\right)$ the mechanical reinforcement ratio $\omega$ and the column slenderness $\lambda$ are obtained.

2. From Eq. 14 and from the relationships reported in Appendix, $\alpha$ and $v_{P E}{ }^{*}$ are respectively calculated, in order to define the limit of the two branches of the domain.

3. If ${v_{S d}}{ }^{*}>v_{P E}{ }^{*}$, the problem is governed by the "parabolic" branch, otherwise it is governed by the "elliptic" one;

4. APPENDIX provides the coefficients $\kappa_{i}$ related to the selected branch;

5. The allowable I-order moment $\mu^{* I}$ is obtained from Eq. 13 or Eq. 16; 
6. The effect of uncertainties related to the geometry and the position of the axial load is calculated by Eq. 12 , providing the reduced moment $\mu_{0}{ }^{{ }^{*} I}$ from;

7. Finally, the effective resistant moment $\mu_{d}{ }^{* I}=\mu^{* I}-\mu_{0}{ }^{* I}$ is obtained.

The approach is particularly interesting because it overcomes some difficulties connected with the use of numerical approaches deduced from other methods. In fact, although conceptually simple, the use of "reduced resistance domains" is often characterised by difficulties and mistakes essentially due to:

- the need of interpolating the values of slenderness and reinforcement not directly reported in the domains;

- the need of a graphical determination of the resistance values.

The major advantage of the procedure is represented by the possibility of analysing in a very simple way the response of isolated R.C. columns (rectangular, constant section and symmetrical reinforcements), loaded by steady longitudinal forces, and characterised by material of any value of resistances, under the only condition that the constitutive laws fulfil those suggested by Eurocode 2.

\section{CONFLICT OF INTEREST}

The author(s) confirm that this article content has no conflicts of interest.

\section{ACKNOWLEDGEMENT}

The research presented in this article was partially funded by the Department of Civil Protection, Project ReLUIS-DPC 2010-2013.

\section{LIST OF SYMBOLS}

\begin{tabular}{|c|c|}
\hline$a$ & $=$ Deflection of the top section of column \\
\hline$A$ & $=$ Transversal area section \\
\hline$A_{\text {bottom, }}$ & $\begin{aligned}= & \text { Total area of steel reinforcement bars at the } \\
& \text { base section }\end{aligned}$ \\
\hline$A_{\text {top }}$ & $\begin{array}{l}=\text { Total area of steel reinforcement bars at the } \\
\text { top section }\end{array}$ \\
\hline$e_{0}$ & $=$ Eccentricity of the applied load \\
\hline$E I$ & $\begin{aligned} &= \text { Effective flexural stiffness of the slender RC } \\
& \text { column }\end{aligned}$ \\
\hline$f_{c d}$ & 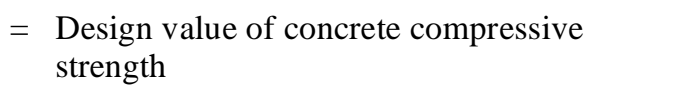 \\
\hline
\end{tabular}

\section{APPENDIX}

The synoptic table (Fig. 11) containing the fundamental relationships required for defining the parabolic-elliptic domain are here explicated.

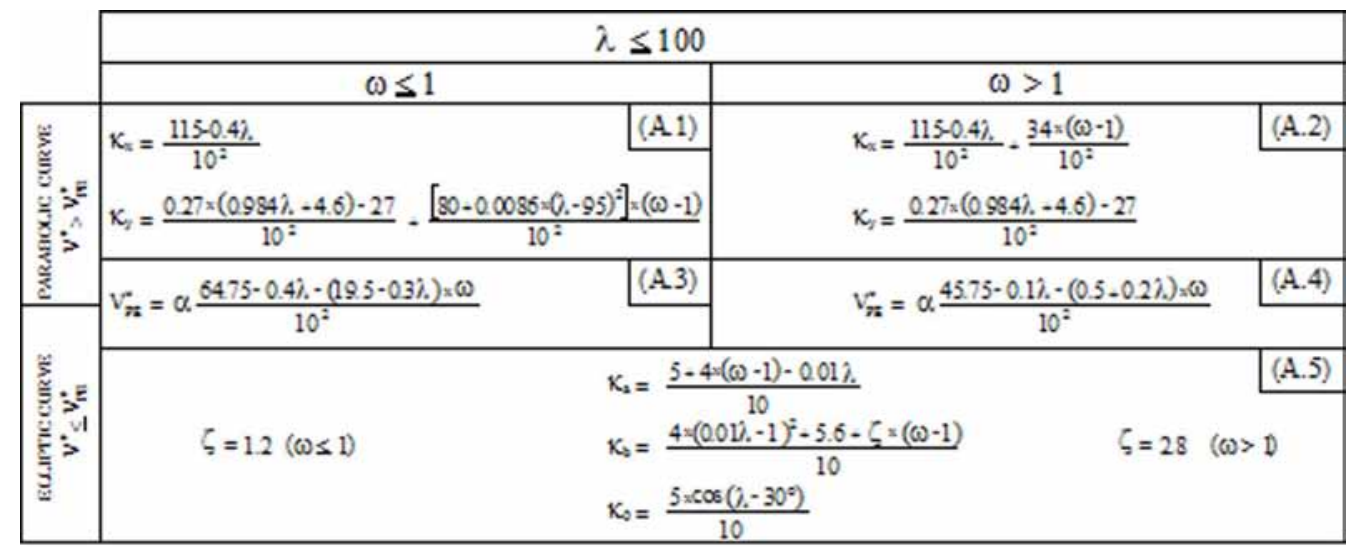

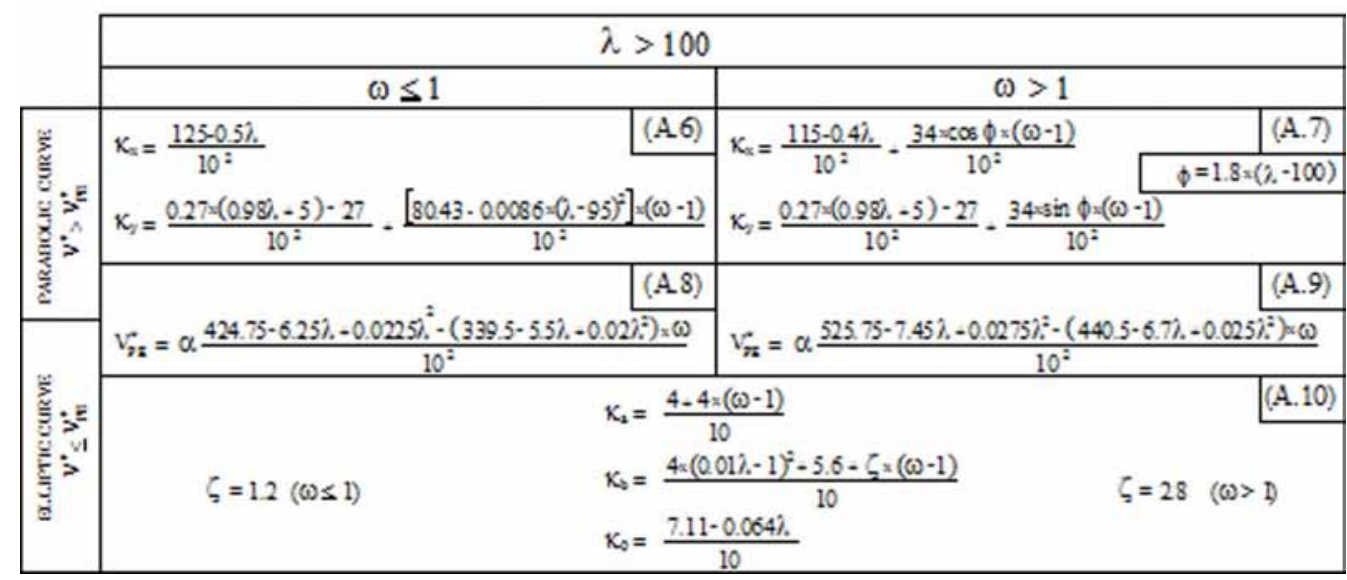

Fig. (11). Synoptic table. 


$$
\begin{aligned}
& f_{y k}=\text { Characteristic yield strength of steel } \\
& i \quad=\text { Radius of inertia } \\
& \text { I }=\text { Moment of inertia of A at the base section of } \\
& \text { the column } \\
& k_{0} \quad=\text { Ordinate value of the center of the ellipse } \\
& k_{a}, k_{b}, k_{a b}=\text { Coefficient values that define the elliptic } \\
& \text { branch of the domain } \\
& k_{x}, k_{y}=\text { Coefficient values that define the parabolic } \\
& \text { branch of the domain. } \\
& L=\text { Column length } \\
& \zeta=\text { Coefficient for assessing the ellipse semi- } \\
& \text { axis } k_{b} \\
& l_{0} \quad=\text { Effective buckling length } \\
& M_{0} \quad=\text { Moment at the base section of the column } \\
& M_{u} \quad=\text { Ultimate moment } \\
& P \quad=\text { Buckling load } \\
& P_{u}{ }^{*} \quad=\text { Maximum load that that the column can sup- } \\
& \text { port } \\
& y(z) \quad=\text { Displacement function along the longitudinal } \\
& \text { axis } \\
& z \quad=\text { Reference abscissa along the longitudinal } \\
& \text { axis of the column } \\
& \alpha=\alpha\left(f_{y k}, \lambda\right)=\text { Scaling factor of the dimensionless axial } \\
& \text { force } v^{*} \\
& \chi_{0} \quad=\text { Curvature of the base section of the column } \\
& \lambda=\text { Column slenderness ratio. } \\
& \mu^{* I}=\text { Dimensionless bending moment of the first } \\
& \text { order } \\
& \mu_{d}{ }^{* I} \quad=\text { Design value of the dimensionless bending } \\
& \text { moment of the first order (i.e. maximum } \\
& \text { available dimensionless I order moment) } \\
& \mu_{0}{ }^{* I}=\text { Dimensionless bending moment of the first } \\
& \text { order at the base section of the column } \\
& v_{p E}{ }^{*}=\text { Dimensionless axial limit force that marks } \\
& \text { the boundary between parabolic and elliptic } \\
& \text { branch } \\
& \omega=\text { Mechanical reinforcement ratio }
\end{aligned}
$$

\section{REFERENCES}

[1] D. Raffaele, F. Porco, A. Fiore, and G. Uva, "Simplified vulnerability assessment of reinforced circular piers in multi-span-simply supported bridges", Structure and Infrastructure Engineering, [E pub ahead of print] 2013.

[2] G. Uva, F. Porco, A. Fiore, and M. Mezzina, "Proposal of a methodology of in-situ concrete tests and improving the estimate of the compressive strength", Construction and Building Materials, vol. 38, no. 1, pp. 72-83, 2013.

[3] A. Fiore, F. Porco, G. Uva, and M. Mezzina, "On the dispersion of data collected by in situ diagnostic of the existing concrete", Construction and Building Materials, vol. 47, pp. 208-217, 2013.

[4] Z. P. Bazant, L. Cedolin, and M. R. Tabbara, "New method of analysis for slender columns", ACI Structural Journal, vol. 88, no. 4, pp. 391-401, 1991.

[5] Z. P. Bazant, and Y. Xiang, "Inelastic buckling of concrete column in braced frame" Journal of Structural Engineering, vol. 123, no. 5 , pp. 634-642, 1997.

[6] J. K. Kim, and S. S. Lee, "The behavior of reinforced concrete columns subjected to axial force and biaxial bending", Engineering Structures, vol. 22, no. 11, pp. 1518-1528, 2000.

[7] J. K. Kim, and J. K. Yang, "Buckling behaviour of slender high strength concrete columns", Engineering Structures, vol. 17, no. 1, pp. 39-51, 1995.

[8] C. Yalcin, and M. Saatcioglu, "Inelastic analysis of reinforced concrete columns", Computer \& Structures, vol. 77, no. 5, pp. 539555, 2000.

[9] CEN. Eurocode 2. Design of Concrete Structures - Part 1-1: General rules and rules of buildings. European Prestandard, EN 19921-1, Brussels, Belgium; 2005.

[10] ACI Committee 318. Building Code Requirements For Structural Concrete. American Concrete Institute: Farmington Hills, MI, 2008, p. 520.

[11] S. A. Mirza, P.M. Lee, and D. L. Morgan, "Stability resistance factor for RC columns", Journal of Structural Engineering, vol. 113, no. 9, pp. 1963-1976, 1987.

[12] S. A. Mirza, "Flexural stiffness of rectangular reinforced concrete columns", ACI Structural Journal, vol. 87, no. 4, pp. 425-435, 1990

[13] J. L. Bonet, P. F. Miguel, M. A. Fernandez, and M. L. Romero, "Biaxial bending moment magnifier method", Engineering Structures, vol. 26, no. 13, pp. 2007-2019, 2004.

[14] T. K. Tikka, and S. A. Mirza, "Nonlinear EI equation for slender reinforced concrete columns", ACI Structural Journal, vol. 102, no. 6, pp. 839-848, 2005.

[15] T. K. Tikka, and S. A. Mirza, "Effective flexural stiffness of slender structural concrete columns", Canadian Journal of Civil Engineering, vol. 35, pp. 384-399, 2008.

[16] J. L. Bonet, M. L. Romero, and P. F. Miguel, "Effective flexural stiffness of slender reinforced concrete columns under axial forces and biaxial bending", Engineering Structures, vol. 33, pp. 881-893, 2011.

[17] H. G. Kwak, and S. P. Kim, "Ultimate resisting capacity of slender RC columns", Computer \& Structures, vol. 82, pp. 901-915, 2004.

[18] N. Krauberger, S. Bratina, M. Saje, S. Schnabl, and I. Planinc, "Inelastic buckling load of a locally weakened reinforced concrete column”, Engineering Structures, vol. 34, pp. 278-288, 2011.

[19] D. Raffaele, "U.L.S. for buckling of isolated reinforced concrete columns low resistance domains", L'industria Italiana del Cemento, vol. 798, pp. 416-439, 2004.

(C) Raffaele et al.; Licensee Bentham Open.

This is an open access article licensed under the terms of the Creative Commons Attribution Non-Commercial License (http://creativecommons.org/licenses/by-nc/3.0/) which permits unrestricted, non-commercial use, distribution and reproduction in any medium, provided the work is properly cited. 